\title{
OPTIMAL MODEL REFERENCE CONTROLLER BASED ON TWO OPTIMIZATION ALGORITHMS FOR MEAN ARTERIAL PRESSURE
}

\author{
${ }^{*}$ Rawaa H. Abed ${ }^{1}$
}

Ekhlass H. Karam²

1) Mustansiriyah University/ Computer Engineering Department, Diyala, Iraq.

2) Mustansiriyah University/ Computer Engineering Department, Baghdad, Iraq.

\begin{abstract}
This paper Presents optimal Model Reference Controller (OMRC) to regulate the high blood pressure. It is based on slate model which was adopted by many researchers. In this work Squirrel Search Algorithm (SSA) and Grey Wolf Optimizer (GWO) algorithms are considered to optimize the controller parameters and compare between its results. the results showed that the suggested controller has good performance and stabilize the mean arterial pressure with small settling time (below than 400s) and small overshoot (below than $5 \mathrm{mmHg}$ ) with low amount of error.
\end{abstract}

Keywords: Blood Pressure; Squirrel Search Algorithm; Grey Wolf Optimizer.

\section{Introduction}

Pressure of the blood is one of the most popular worldwide diseases afflicting humans and is a significant risk factor for stroke, myocardial infarction, vascular disease, and chronic kidney disease. two types of blood Pressure; high blood pressure (hypertension) and low blood pressure (hypotension), it is measured in millimeters of mercury unit ( $\mathrm{mmHg}$ ). the complication become less when the blood pressure of the patients is controlled [1]. Controlling the MAP (Mean Arterial Pressure) is consider one of the problems that must be controlled during surgery by anesthesiologist. The procedure of the MAP lowering can be done by injecting the patient body by low pressure medications like Sodium Nitroprusside (SNP) [2]. Sodium nitroprusside is a powerful anti-hypertension medicine that is only used in when blood pressure should be reduced immediately.

For example, patients with high blood pressure after cardiovascular surgery may be given sodium nitroprusside, it is causing the muscles around the blood vessels to relax, allowing the blood vessels to wide, as the blood vessels dilate, blood pressure decreases [3]. In recent and past years, some researchers have tried to design a blood pressure control device during surgery to reduce to relieve the shoulders of work on anesthesiologists by quantifying the amount of medication that a patient should be entitled in a smart way [4].

So, an automatic drug infusion system is helpful efficient the control of drug infusion in a proper way, the patient injection system is an integrated system contains of injection materials as per the medical standards, which will be sensing the blood pressure level and determine the amount of drug required to injecting the human body with the help of automatic pump [5]. In recent years,

* Corresponding author: engrawaa1990@gmail.com 
several researchers developed adaptive control system to regulate the rising of blood pressure like Fractional Order Adaptive Regulator (FOAR) which is suggested by Samir Ladaci et al. [6]. The proposed adaptive controller gave the stability and a good level of durability against turbulence for MAP system. Humberto A. Silva1 et al. [8] use the Multi-Model Adaptive Control (MMAC) to regulate the MAP, The results showed that the multi-model schema has great potential for application in unconfirmed systems, and it gave punished results even with noise. Anderson Luiz et al. [7] use an Adaptive Predictive Controller (APC) and a Proportional Integral (PI) controller with Fuzzy system consideration of two patients simulated, an advantage of this suggested controller is that no need for tuning parameters set by the user, unlike other controllers. However, its implementation is more difficult and its effort is higher in comparison with other controllers. Simple adaptive PI controller suggested by Samuel Justino da Silva1 et al. [9]. For mean arterial pressure regulation the controller is meets the required system standards in terms of: settling time less than $600 \mathrm{~s}$, overshoot less than 10 $\mathrm{mmHg}$, and steady-state error about setpoint less than $5 \mathrm{mmHg}$. All of these controllers are adaptive controllers, although it gave good results, it is somewhat complicated and difficult to implemented. So, in this paper, Optimal Model Reference controller (OMRC) is suggested instead of Model Reference Adaptive Controller (MRAC) to regulate the mean arterial pressure with less effort and easy implementation as comparison with other controllers, based on the Squirrel Search Algorithm (SSA) and Grey Wolf Optimizer (GWO) algorithm to tune its parameters. Different mathematical models that clarifies relationship between blood pressure and vasoactive drug injection have been investigated since the 1980 s, one of them is the Slate model which considered here. The remaining article is consisting of: section 2 discussed the mathematical model of MAP. Section 3 describes the OMRC controller, while section 4 illustrates the SSA and GWO algorithms. Section 5 contain the results of simulation and comparison between
SSA and GWO algorithms. Section 6 is the final conclusion section.

\section{Mean Arterial Pressure Modulization.}

The most common used mathematical model of the mean arterial pressure (MAP) of a patient and very popular introduced in 1980 by slate $[10,11]$ after more studies on patients which leaded to the following mathematical equation:

$\operatorname{MAP}(\mathrm{t})=\Delta \mathrm{pd}(\mathrm{t})+\mathrm{p}_{0}$

MAP is the mean arterial blood pressure, $\Delta p d(t)$ is the change in blood pressure after injected the patient with SNP given in $\mathrm{mmHg}, \quad p_{0}$ is the initial blood pressure. The transfer function of $\Delta p d(t)$ relating to SNP infusion rate is [12]:

$$
\frac{\Delta p d(s)}{I(s)}=\frac{K e^{-T i s}\left(1+\alpha e^{-T c s}\right)}{1+T s}
$$

where $I(s)$ is infusion rate, $K$ is the drug sensitivity, $\alpha$ is the drug fraction recirculation, $T i$ is the initial transport lag from injection site, $T c$ is the time consuming by the drug to flow through the patient's body, $T$ is the time required for distribution and biotransformation of the drug $[13,14]$.

\section{Optimal Model Reference Controller (OMRC)}

The standard MRAC include two loops, the internal loop (adjustment of parameter loop) and the external loop (feedback loop).This controller usually works based on adjusting the control parameters such that the actual output of the plant follow the output of the desired reference model that has the same input reference signal [15].

In this paper, an Optimal Model Reference Controller (OMRC) is suggested, where the 
parameters of this controller are optimized using SSA and GWO algorithms (using Squared error cost function it is simplest function and also ensures that the errors are always positive), while being such that ccontroller accomplishes the required response. Figure. 1 present the patient's model and OMRC controller diagram. The control signal up represents the rate of infusing drug which is a linear combination of the error feedback $K_{d} I_{d}$,reference model output $K_{m} I_{m}$ and reference model input $K_{e} I_{e}$. The law of the adaptive controller involve the values of the reference model output " $I_{m}$ ", tracking error " $I_{d}$ ", and the reference model input" $\mathrm{I}_{\mathrm{e}}$ " with a suitable Optimal gains $(K e, K m$, and $K d$ ) which optimize with SSA and GWO algorithms that are illustrated in the section 4 . The optimal control Equation is given by [16]:

$u_{p}(t)=K_{e} I_{e}(t)+K_{m} I_{m}(t)+K_{d} I_{d}(t)$

the model reference is selected by the user according to the mathematical model in this work the model reference is same to the transfer function of the patient model without any delay: $m(s)=\frac{1}{\mathrm{G} \mathrm{s}+1}$

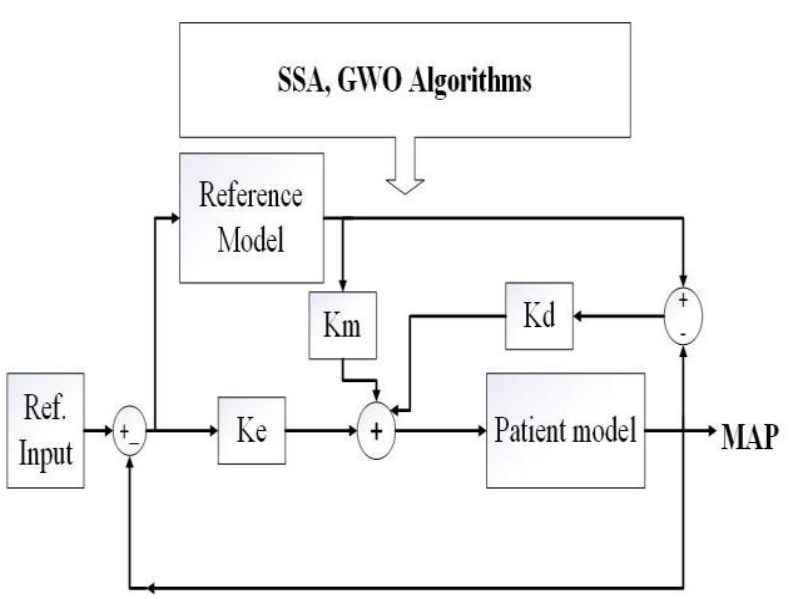

Figure 1. OMRC diagram.

\section{Optimization Algorithms}

\subsection{The Squirrel Search Algorithm}

This algorithm (SSA) Suggested et al. in 2019 by Mohit Jain. it was a novel nature-inspired algorithm for optimization, (SSA) algorithm consist of four the search processes of flying squirrels: (i). there are $P$ flying squirrels and $P$ trees and only one squirrel on one tree, (ii). each flying squirrel is tries to find the food so each one searching for food individually, (iv). there are only three types of trees such as normal tree, oak tree and hickory tree (which is the favor tree) in forest only three oak trees and one hickory tree in the forest [17]. The following steps explained the operation of this algorithm [18]

Step 1: Initialize parameters of the SSA algorithm; $P$ the population nomber, Gen the ateration nomber, $F_{U}, F_{L}$ are lower and upper bounds, $P d_{p}$ the presence of predators, $R_{1}, R_{2}$, $R_{3}$ is 1 or 0 (Predator presence probability $P d_{p}$ ) is considered to be 1 in all cases for the present work, $\rho=1.204 \mathrm{Kgm}^{-3}$ is density of air, $C L$ is called as lift coefficient, $V=5.25 \mathrm{~ms}^{-1}$ is speed and $S=154 \mathrm{~cm} 2$ is the surface area of body [18], clculate $d_{g}, D, L, \tan (\Phi)$ according to the Equations :

$$
\begin{aligned}
& d_{g}=\frac{h_{g}}{\tan \Phi} \\
& \tan (\Phi)=\frac{D}{L} \\
& \mathrm{D}=\frac{1}{2 p v s C D} \\
& L=\frac{1}{2 p v s C L}
\end{aligned}
$$

$d_{g}$ is the gliding distance, $h_{g}$ the constant, tan $(\Phi)$ is the angle of gliding. 
Step2: evaluate the fitness value value and classify the population.

Step3: If there is no predator update the position of squarrls which are on the ocorn trees moving towards hickory tree According to the winter updating strategiah:

$\mathrm{fso}(\mathrm{t}+1)=\mathrm{fso}(\mathrm{t})+\mathrm{dg}$ Gc $(\mathrm{fsh}-\mathrm{fso})$

where $f$ so squarrls which are on the ocorn trees, $f s h$ squarrls which are on the hickory trees

Else

Random location

Step4: If there is no predator update the position of squarrls which are on the normal trees moving towards ocorn tree According to the winter updating strategiah.

$$
\begin{aligned}
f \operatorname{sn}(t+1)= & f s n(t) \\
& +d g G c(f s o-f s n)
\end{aligned}
$$

where $f s o$ squarrls which are on the ocorn trees, $f s n$ squarrls which are on the normal trees

Else

Random location

Step5: If there is no predator update the position of squarrls which are on the normal trees moving towards hickory tree According to the winter updating strategiah:

$$
\begin{aligned}
f \operatorname{sn}(t+1)= & f \operatorname{sn}(t) \\
& +d g G c(f s h-f s n)
\end{aligned}
$$

Else

Random location

Step 6: If the winter season is over updating the position of all squirrels according to the summer strategies: $\mathrm{fs}_{\mathrm{n}, \mathrm{h}, \mathrm{o}}(\mathrm{t}+1)=F_{L}+\mathrm{Le}^{\prime} \mathrm{vy}(\mathrm{n})\left(F_{U}-F_{L}\right)$

$\operatorname{Le}^{\prime} \operatorname{vy}(n)=0.01 \frac{r_{a} \sigma}{\left|r_{b}\right|^{1 / \beta}}$

$\sigma=\left(\frac{\Gamma(1+\beta) \sin \frac{\beta \pi}{2}}{\Gamma\left(\frac{1+\beta}{2}\right) \beta 2^{\frac{\beta-1}{2}}}\right)^{\frac{1}{\beta}}$

where $\Gamma(x)=(x 1)$ !

Fig. 2 illustrates the procedure of SSA algorithm.

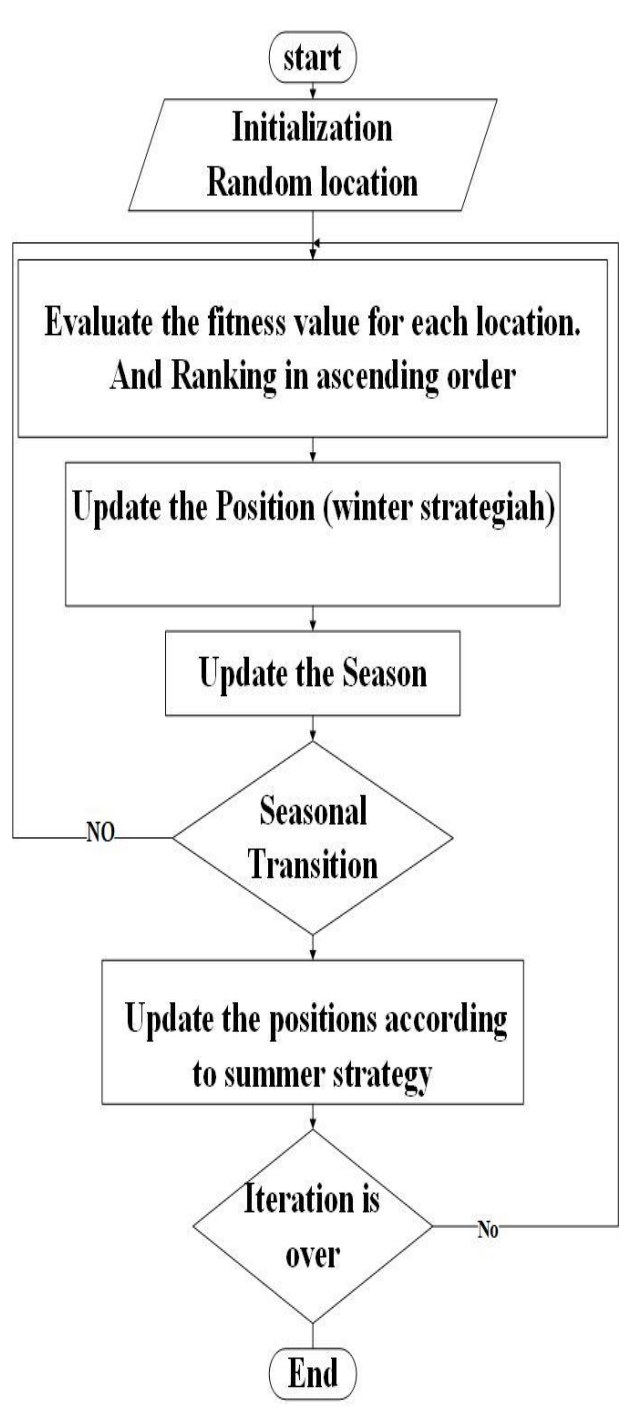

Figure 2. SSA Procedure. 


\subsection{Grey Wolf Optimizer}

Mir Jalili proposes the GWO algorithm in 2014. It is suggested in order to find prey According to the Gray wolf social hierarchy Hunting practices are the solution to the problem of optimization. The social hierarchy is represented by splitting the quest agent population into four groups of wolves, i.e., alpha, beta, delta, and omega, based on their fitness. In order to mimic the hunting actions of grey wolves, the quest technique is modelled using three stages that encircle and attack the prey [19].

The GWO comprises the steps established by the revision of the measures [20]:

Step 1. The initial random grey wolf population, represented by $P$ agents' positions in the $D$ dimensional search space, is generated. The iteration index is initialized to $t=0$ and the number of iterations is set to Gen.

Step 2. Evaluated the fitness value for each wolf based on the least-square error (LSE) criterion

Step 3. the first three best solutions $z^{\alpha}, z^{\beta}, z^{\delta}$, which represented alpha, beta, delta positions.

Step 4. The agents are moved to their new positions according to the Equation below.

$Z_{\text {new }}=\frac{\left(c_{1}+c_{2}+c_{3}\right)}{3}$

$c_{1}, c_{2}, c_{3}$ calculated from equations below

$c_{1}=z^{\alpha}-A_{1} D_{\text {alpha }}$

$c_{2}=z^{\beta}-A_{2} D_{\text {beta }}$

$c_{3}=z^{\delta}-A_{3} D_{\text {delta }}$

$A_{1}, A_{2}, A_{3}=2 a r_{1}-a$

$r_{1}$ is random value having different value for

$A_{1}, A_{2}, A_{3}, a$ is Variable value.

$a=2-t\left(\frac{2}{T}\right)$
$D_{\text {alpha }}, D_{\text {beta }}, D_{\text {delta }}$ are computed from equations below.

$\mathrm{D}_{\text {alpha }}=\operatorname{abs}\left(\mathrm{m}_{1} \mathrm{z}^{\alpha}-\mathrm{z}_{\text {current }}\right)$

$\mathrm{D}_{\text {beta }}=\operatorname{abs}\left(\mathrm{m}_{2} \mathrm{z}^{\beta}-\mathrm{z}_{\text {current }}\right)$

$\mathrm{D}_{\text {delta }}=\operatorname{abs}\left(\mathrm{m}_{3} \mathrm{z}^{\delta}-\mathrm{z}_{\text {current }}\right)$

$m_{1}, m_{2}, \mathrm{~m}=2 r_{2}$

$r_{2}$ is random value having different value for $m_{1}, m_{2}, m_{3}$.

Step 5. If all iteration will be finished, stop the search and display the first three best solution e Otherwise, repeat step (2) to step (4) before iterations have been finalized.

Fig. 3 illustrates the procedure of the GWO

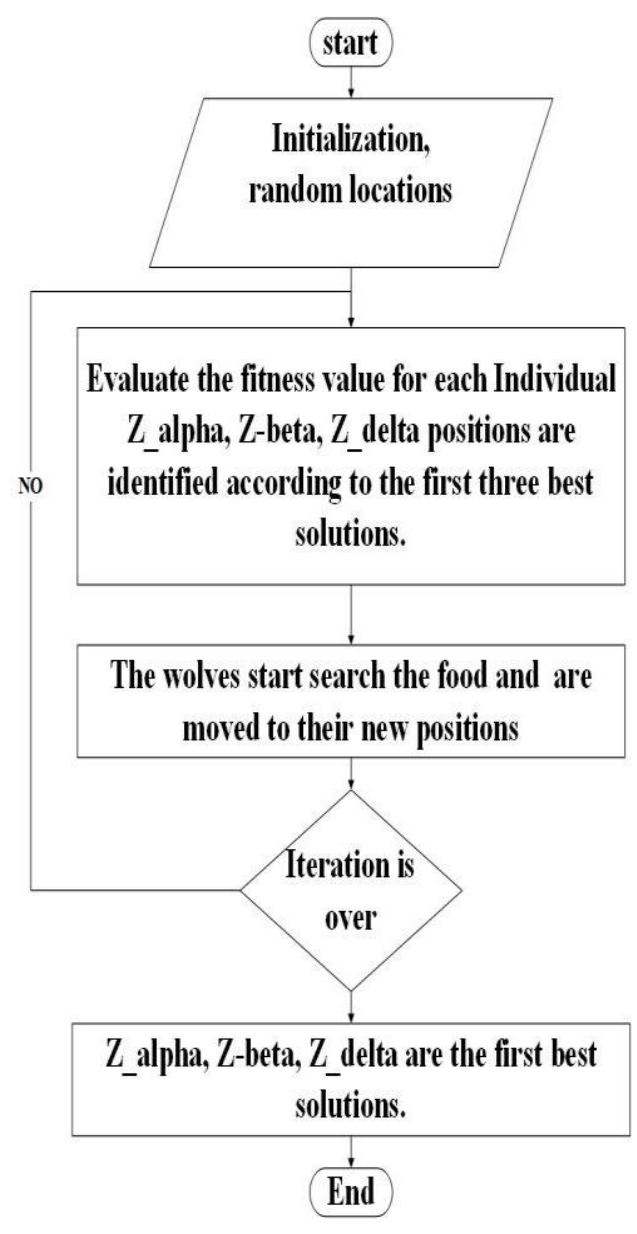

Figure 3. GWO Procedure. 


\section{Simulation and Results}

The simulation results of the suggested controller based on the SSA, GWO algorithms for controlling MAP regulation model have been presented in this section. The control simulated with patient model using MATLAB program. The initial value of the patient MAP is chosen as $140(\mathrm{mmHg})$, The control objective is to reduce the MAP to $100(\mathrm{mmHg})$ for three cases (sensitive patient, normal patient, insensitive patient) without disturbance, the parameters of MAP model illustrated in Table (1). The parameters SSA and GWO are considered in this Table (2) Shown. The simulation response of MAP model after regulated it by the suggested controller (OMRC) which tuned by the SSA and GWO algorithms as illustrated in Table (3) is shown in Fig. 4 and Fig. 5 respectively. Fig. 6 and Figure (7) show the controller signal.

Table 1. model Parameters [21].

\begin{tabular}{lrrr}
\hline parameters & SEN & NOR & INS \\
$\mathrm{Ti}$ & 20 & 30 & 60 \\
$\mathrm{~K}$ & -9 & -0.7143 & -0.1786 \\
$\mathrm{~T}$ & 30 & 40 & 60 \\
$\mathrm{Tc}$ & 30 & 45 & 75 \\
\hline
\end{tabular}

Table 2. The SSA and GWO algorithms parameters.

\begin{tabular}{lclc}
\hline SSA parameters & value & GWO parameters & value \\
Search agent no $(\mathrm{P})$ & 25 & Search agent no $(\mathrm{N})$ & 25 \\
Max iteration $(\mathrm{Gen})$ & 15 & Max iteration $(\mathrm{T})$ & 20 \\
Dimension $(\mathrm{D})$ & 3 & Dimension $(\mathrm{D})$ & 3 \\
Upper limit $\left(\mathrm{F}_{\mathrm{L}}\right)$ & 0.75 & --- & --- \\
Lower limit $\left(\mathrm{F}_{\mathrm{U}}\right)$ & 0 & --- & --- \\
$\begin{array}{l}\text { predator appearance } \\
\left(\mathrm{pd}_{\mathrm{p}}\right)\end{array}$ & 1 & --- & --- \\
\hline
\end{tabular}


Table 4. The evaluation parameters of simulation results for three cases.

\section{SSA algorithm}

\begin{tabular}{lccc}
\hline \multicolumn{1}{c}{ Case } & $\mathbf{K}_{\mathbf{e}}$ & $\mathbf{K}_{\boldsymbol{m}}$ & $\mathbf{K}_{\boldsymbol{d}}$ \\
Sensitive & -0.0892 & 0.1150 & -0.0444 \\
Normal & 0.58422 & -0.00026 & -0.4011 \\
Insensitive & -2.2593 & 3.4332 & -1.6111 \\
\hline GWO algorithm & & & \\
\hline Case & $\mathbf{K}_{\mathbf{e}}$ & $\mathbf{K}_{\boldsymbol{m}}$ & $\mathbf{K}_{\boldsymbol{d}}$ \\
Sensitive & -0.0055 & -0.0055 & -0.0444 \\
Normal & 0.1120 & 0.2356 & -0.4001 \\
Insensitive & -0.0015 & 1.01131 & -1.6011 \\
G & 50 for all cases (the parameter of the model reference) \\
\hline
\end{tabular}

Table (4) also illustrates that the performance of the suggested (OMRC) optimized by SSA algorithm is nearly similar to the performance of controller when optimized by GWO algorithm.

Table 4. The evaluation parameters of simulation results for three cases.

\begin{tabular}{lrlrrr}
\hline \multicolumn{1}{c}{ SSA Algorithm } & \multicolumn{5}{c}{} \\
\hline \multicolumn{1}{c}{ Case } & $\mathbf{M}_{\mathbf{u}}(\mathbf{m m H g})$ & \multicolumn{1}{c}{$\boldsymbol{t}_{\boldsymbol{s}(\boldsymbol{e s c})}$} & $\boldsymbol{t}_{\boldsymbol{d}}(\mathbf{s e c})$ & $\boldsymbol{e}_{\boldsymbol{s s}}(\mathbf{m m H g})$ & $\mathbf{S N P}(\mathbf{m l} / \mathbf{h})$ \\
Sensitive & 4.3215 & 115.3596 & 16.8050 & 0.0255 & 4.441 \\
Normal & 4.6047 & 324.1092 & 77.732 & 0.0771 & 40.09 \\
Insensitive & 4.2969 & 371.4888 & 75.0828 & 0.0981 & 160.09 \\
\hline GWO Algorithm & & & & & \\
\hline Sensitive & 4.6931 & 173.7755 & 24.7003 & 0.0215 & 4.442 \\
Normal & 3.5761 & 229.2142 & 50.1251 & 0.0042 & 40.01 \\
Insensitive & 4.5039 & 329.1991 & 85.7126 & 0.0651 & 160.3 \\
\hline
\end{tabular}


It is obvious from Fig. 4 and Fig. 5 that the controller satisfies the design requirement by making the MAP system follows the desired level with small steady state error $\left(e_{s s}\right)$, settling time $\left(t_{s}\right)$, drop time $\left(t_{d}\right)$ and undershoot $\left(M_{u}\right)$ as shown in Table (4).

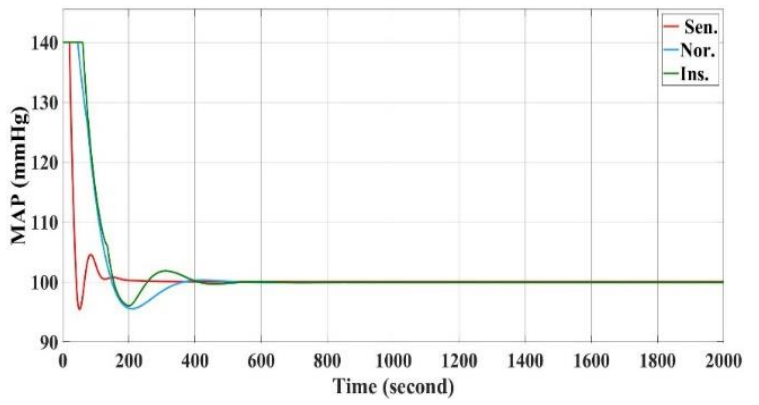

Figure 4. The response of MAP model according to the OMRC optimized by SSA algorithm.

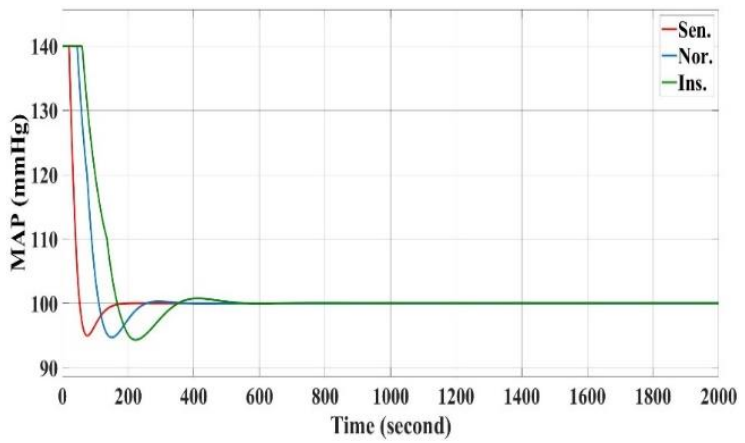

Figure 5. The response of MAP model according to the OMRC optimized by GWO algorithm.

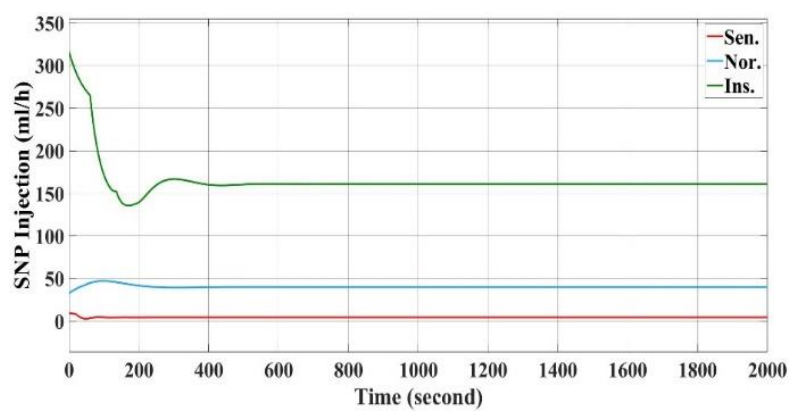

Figure 6. SNP injection according to the OMRC optimized by SSA algorithm.

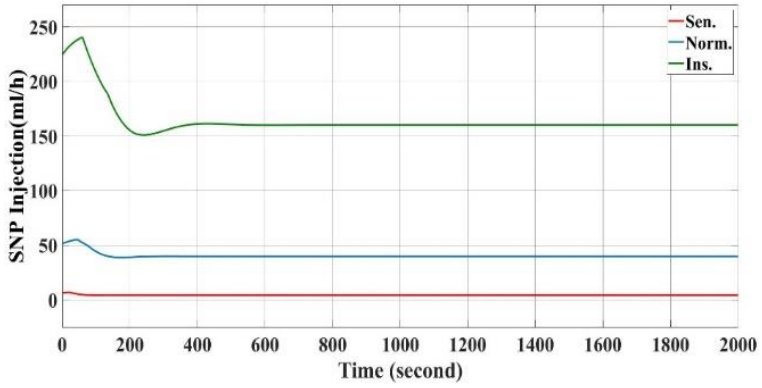

Figure 7. SNP injection according to the OMRC optimized by GWO algorithm
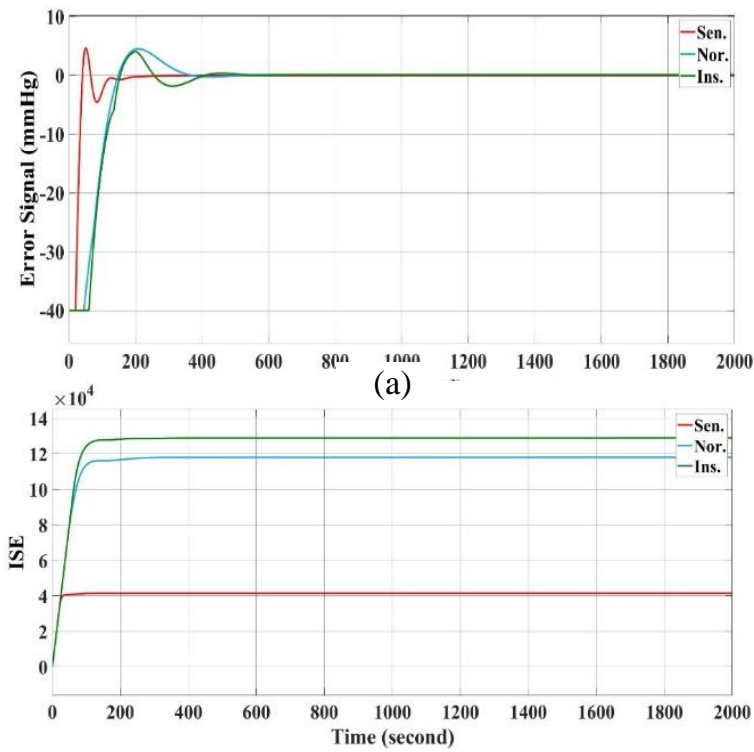

(b)

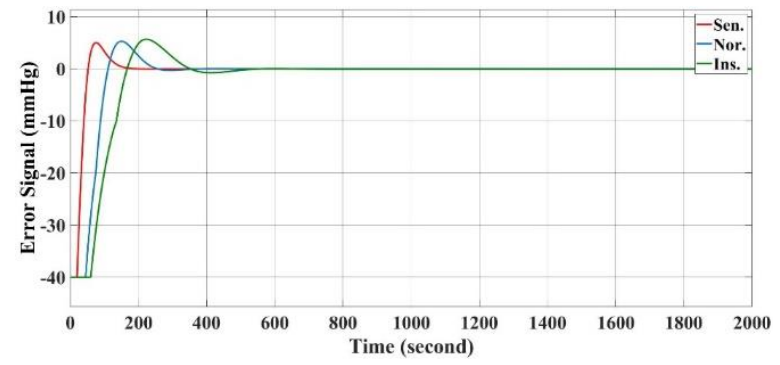

(c)

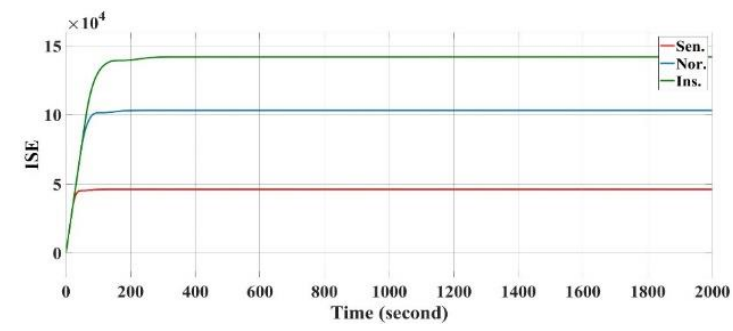

(d)

Figure 8. (a) Error signal (SSA), (b)ISE signal (SSA), (c) Error signal (GWO), (d) ISE signal (GWO). 
As can be seen from the Fig. 4,5 and Table (4) above, the response varies from patient to patient due to the different sensitivity of each patient to the drug, for example a patient who has a great sensitivity to the drug, the response speed of the system is very high and vice versa patient, whose sensitivity to the drug is low, , the undershoot is no more than $(5 \mathrm{mmHg})$ this value is accepted according to the slate criteria [12].

\section{Conclusion}

The paper has presented an optimal single-drug control scheme for MAP control. The suggested controllers designed and evaluated by simulation results for different variations in patients (normal, sensitive, and insensitive). In this paper single input single output (SISO) system is used to control the mean arterial pressure using single drug like nitroprusside (SNP). The results of the simulation have shown that OMRC is more efficient in regulating the MAP by calculated the infusion rates of the SNP. In order to improve the characteristics of the controller, SSA, GWO algorithms have been applied. For future work we suggest use same controller using adaptive adjustment mechanism for tuning the parameters of the controller such as Neural Network to regulate mean arterial pressure the controller became intelligent control and more efficient to control this type of model (dynamic model).

\section{Acknowledgements}

All thanks and appreciation to those who helped me and gave me scientific advice in this research

\section{Conflict of interest}

the publication of this article causes no conflict of interest.

\section{References}

1. B. Singh and S. Urooj. (2019). "Blood pressure control by deterministic learning based fuzzy logic control," Int. J. Eng. Adv. Technol., vol. 8, no. 3, pp. 6-10.

2. Á. Herrero et al.(2014). "Mean Arterial Pressure PID Control Using a PSO-BOIDS Algorithm," Adv. Intell. Syst.Comput., vol. 239, pp. v-vi.

3. N. Malagutti, A. Dehghani, and R. A. Kennedy. (2013). "Robust control design for automatic regulation of blood pressure," IET Control Theory Appl., vol. 7, no. 3, pp. 387396.

4. A. G. C. D. Ribeiro et al. (2011) "Design of an embedded PID controller applied to blood pressure control,’ Proc. Annu. Int. Conf. IEEE Eng. Med. Biol. Soc. EMBS, no. August, pp. 278-281.

5. A. A. Basha, S. Vivekanandan, and P. Parthasarathy. (2018). "Evolution of blood pressure control identification in lieu of postsurgery diabetic patients: a review," Heal. Inf. Sci. Syst., vol. 6, no. 1.

6. S. Ladaci. (2012). "Postoperative Blood Pressure Control Using a Fractional order Adaptive Regulator," pp. 254-265.

7. A. Luiz, D. E. O. Cavalcanti, and A. L. Maitelli. (2015). "Design of an Intelligent Adaptive Drug Delivery System for Arterial Pressure Control," vol. 10, pp. 704-712.

8. H. A. Silva, C. P. Leão, and E. A. Seabra. (2018). "Parametric Sensitivity Analysis of a Multiple Model Adaptive Predictive Control for Regulation of Mean Arterial Blood Pressure," vol. 1, no. Icinco, pp. 520-526.

9. S. J. da Silva, T. A. Scardovelli, S. R. M. da Silva Boschi, S. C. M. Rodrigues, and A. P. da Silva. (2019). "Simple adaptive PI controller development and evaluation for mean arterial 
pressure regulation," Res. Biomed. Eng., vol. 35 , no. 2, pp. 157-165.

10. J. B. Slate and L. C. Sheppard. (1983). "Model-Based Adaptive Blood Pressure Controller.," IFAC Proc. Vol., vol. 2, no. 4, pp. 1437-1442.

11. S. Saxena and Y. V. Hote. (2012). "A simulation study on optimal IMC based PI/PID controller for mean arterial blood pressure,” Biomed. Eng. Lett., vol. 2, no. 4, pp. 240-248.

12. A. A. Basha and S. Vivekanandan. (2019)."Enhanced Optimal Insulin Regulation in Post-Operative Diabetic Patients: An Adaptive Cascade Control CompensationBased Approach With Diabetic and Hypertension," IEEE Access, vol. 7, no. Cvd, pp. 90973-90981.

13. S. Urooj and B. Singh. (2019). "Fractionalorder PID control for postoperative mean arterial blood pressure control scheme," Procedia Comput. Sci., vol. 152, pp. 380-389.

14. R. W. Jones and M. T. Tham. (2005). "An undergraduate CACSD project: The control of mean arterial blood pressure during surgery," Int. J. Eng. Educ., vol. 21, no. 6 PART I, pp. 1043-1049.

15. P. Jain and N. M.J. (2013) "Design of a Model Reference Adaptive Controller Using Modified MIT Rule for a Second Order System," Adv. Electron. Electr. Eng., vol. 3, no. 4, pp. 477-484.
16. S. Enbiya, F. Mahieddine, and A. Hossain.(2011) "Model reference adaptive scheme for multi-drug infusion for blood pressure control.," J. Integr. Bioinform., vol. 8, no. 3, p. 173.

17. H. Hu, L. Zhang, Y. Bai, P. Wang, and X. Tan. (2019). "A Hybrid Algorithm Based on Squirrel Search Algorithm and Invasive Weed Optimization for Optimization," IEEE Access, vol. 7, pp. 105652-105668.

18. Y. Xiaobing, Y. Xianrui, and C. Hong. (2019). "An improved gravitational search algorithm for global optimization," J. Intell. Fuzzy Syst., vol. 37, no. 4, pp. 50395047.

19. R. E. Precup, R. C. David, A. I. SzedlakStinean, E. M. Petriu, and F. Dragan. (2017). "An easily understandable grey wolf optimizer and its application to fuzzy controller tuning," Algorithms, vol. 10, no. 2, pp. 1-15.

20. S. Mirjalili, S. M. Mirjalili, and A. Lewis. (2014). "Grey Wolf Optimizer," Adv. Eng. Softw., vol. 69, pp. 46-61.

21. S. A. Nirmala, R. Muthu, and B. Veena Abirami. (2014). "Model Predictive Control of Drug Infusion System for Mean Arterial Pressure Regulation of Critical Care Patients," Res. J. Appl. Sci. Eng. Technol., vol. 7, no. 21, pp. 4601-4605. 